\title{
MULTIPLE ANTENATALLY DIAGNOSED CLASSIC DUPLICATION CYSTS WITH A CONCURRENT COMPLETELY ISOLATED DUPLICATION CYST (CIDC) - A RARE COEXISTENCE
}

\author{
Rajalakshmi B. R¹, Asha Mahadevappa², Anil kumar M.G³ ${ }^{3}$ Sunila4, Manjunath G.V5
}

\section{HOW TO CITE THIS ARTICLE:}

Rajalakshmi B. R, Asha Mahadevappa, Anil kumar M. G, Sunila, Manjunath G. V. "Multiple antenatally diagnosed classic duplication cysts with a concurrent completely isolated duplication cyst (CIDC) - A Rare Coexistence". Journal of Evolution of Medical and Dental Sciences 2013; Vol2, Issue 32, August 12; Page: 6066-6071.

\begin{abstract}
Alimentary tract duplications are uncommon congenital anomalies. A case of male neonate referred with antenatally diagnosed cystic lesions in the thorax and abdomen is presented, which represents a rare example of coexistence of ileal and esophageal duplication cysts with a concurrent Completely Isolated Duplication Cyst (CIDC). Awareness of varied presentations may help in appropriate management during surgery.
\end{abstract}

KEYWORDS: Duplication cyst, completely isolated duplication cyst, enterogenous cyst

INTRODUCTION: Gastrointestinal tract duplications are cystic or tubular structures occurring anywhere from tongue to anus. By definition, they are located in or adjacent to the wall of the gastrointestinal tract (GIT), have smooth muscle in their walls and are lined by alimentary tract mucosa (1, 2). CIDC are an extremely rare variety of gastrointestinal duplications with no discernible communication or connection with the adjacent alimentary tract along with typical histopathologic features of a duplication cyst $(3,4,5)$. Coexistence of CIDC with classic enterogenous duplication cyst has been reported only twice in the literature (3).The present case with antenatal diagnosis of multiple intrathoracic and intraabdominal cystic lesions is reported to highlight the postnatal assessment of these lesions and the surgical management.

CASE REPORT: A full term male neonate was referred with a third trimester antenatal ultrasonographic diagnosis of a large cystic lesion in the abdomen. Baby was asymptomatic till 3 months of age and presented with a large palpable abdominal mass and refusal of feeds for 4 days. Clinically, abdomen was distended with a palpable cystic mass in the right upper quadrant extending to the left side.

The CT scan revealed a cystic mass in the abdomen measuring $9.5 \times 8.5 \times 7.6 \mathrm{~cm}$ in the right upper and lower quadrant causing compression and displacement of adjacent bowel loops and was seen communicating with a cyst in the left lower quadrant measuring $6.5 \times 3.5 \mathrm{~cm}$ (Fig 1a). Two more hypodense cystic lesions were noted, one inferior to the distal body of pancreas measuring $2.2 \times 1.6$ $\mathrm{cm}$ and the other in posterior mediastinum measuring $1.9 \times 2.0 \mathrm{~cm}$ in the right paravertebral gutter at D4-D5 level (Fig 1b) .There was no evidence of communication with the spinal canal/bony defects. A diagnosis of multiple enteric duplication cysts was offered with the differential diagnosis of lymphangiomas /bilobed mesenteric cyst.

A first stage laparotomy was performed, where a large dumbbell shaped cyst measuring $10 \times 6.5 \times 6 \mathrm{~cm}$ in the right hypochondrium extending to the left iliac fossa communicating through the small bowel mesentery adjacent to the ileum was noted (Fig 1c). Surface was congested with blood 
supply from the branches of mesenteric vessels which were dissected and the entire cyst was excised, which exuded $300 \mathrm{ml}$ of mucoid fluid. A small cyst measuring $2 \times 1.5 \mathrm{~cm}$ was identified inferior to the tail of the pancreas which was completely isolated with no connection or communication with the adjacent alimentary tract and was excised. Histopathology of the larger cyst showed a cyst lined by gastric mucosa with parietal and chief cells, submucosa, muscular and serosal layers which proved to be an ileal duplication cyst with ectopic gastric mucosa (Fig 2a\&Fig 2b). The smaller CIDC also showed ectopic gastric mucosa with pseudostratified ciliated columnar respiratory epithelium, submucosa, muscular and serosal layers (Fig 2c).

An elective thoracotomy was planned at seven months of age for removal of the intrathoracic cyst measuring $2 \times 1.5 \mathrm{~cm}$ in the right paravertebral gutter adjacent to esophagus with attachment to the serosal layer of esophagus. Microscopy revealed an esophageal cyst lined by stratified squamous epithelium, respiratory epithelium and gastric mucosa (Fig 2d). The postoperative period was uneventful.

DISCUSSION: Alimentary tract duplications were first described by Wendel in 1911(5) with an incidence of 1:4500 autopsies (5, 6).The main locations are ileum (50\%), esophagus (25\%), colon (15\%), stomach (5\%), and duodenum (4\%) ${ }^{(7) . O n e ~ t h i r d ~ o f ~ s m a l l ~ b o w e l ~ d u p l i c a t i o n s ~ a r e ~}$ symptomatic in neonatal period(2).Duplications are located in or adjacent to the wall of the gastrointestinal tract and share a common blood supply from the adjacent mesenteric vessels $(3,4,8)$ .They can be cystic or tubular, cystic duplications rarely communicate with the intestinal lumen. In contrast, tubular duplications usually communicate with the intestinal lumen (5). All the three duplications in the present case were cystic with no communication with the gut lumen.

Completely isolated duplication cysts are an extremely rare variety of GI duplications with their own blood supply with no connection or communication with the bowel segment $(3,4,5)$.These usually present as an incidental finding on antenatal USG or in the perinatal period (3).The present case had a CIDC in the sub pancreatic region with no discernible connection with the adjacent structures or the GIT. The unique feature of the present case is the coexistence of classic enterogenous cysts i.e. ileal and esophageal cysts with a CIDC, a finding reported only twice in the literature (3).

Duplications usually share a common muscular wall and blood supply with the adjacent intestine. This however, is not the case with thoracic duplications which may lie adjacent or distant from the esophagus without sharing a common muscular wall, as in the present case where it was attached to the serosal layer only (9).Foregut duplication cysts when associated with vertebral anomalies, they are called neurenteric cysts, the other association being malrotation ${ }^{(4,9)}$.The present case had no vertebral abnormalities or malrotation.

Patients who have one duplication are found to have another $(1,3,6)$ and $20 \%$ of intrathoracic foregut duplications are associated with concomitant intraabdominal duplication cysts ${ }^{2}$, 9).This necessitates the need for investigations such as CT scan and careful search during laparotomy, for detection of concurrent cysts.

Ectopic mucosa representing any part of the intestine can be found in GI duplications, most common being gastric mucosa, seen in $50 \%$ cases making it liable to perforate and bleed $(1,3,6,9)$.All the three cysts including the CIDC in our case showed gastric mucosa .The esophageal cysts can be 
lined by epithelium resembling gastric, esophageal and bronchogenic mucosa, all of which were seen in our case $(6,7)$.

Many possible embryological mechanisms have been proposed for the origin of enteric duplications, namely partial or abortive twinning (2,3), split notochord theory (1-3, 8, 9), diverticular and canalization defects $(1,3,9)$ and intrauterine vascular accidents (2,3).Partial or abortive twinning can explain doubling or duplication anomalies of hindgut, and the lower genitourinary tract. The split notochord theory is the most accepted which proposes that abnormal separation of the notochord from gut endoderm can cause dorsal enteric duplications and is the explanation for neurenteric cyst. The theory of aberrant luminal recanalization explains duplications in those portions of the gastrointestinal tract that go through the solid stage during embryonic development such as the esophagus, small intestine, and colon $(2,3,8)$. As such, no single mechanism can account for all the known variants of enteric duplications. In our patient, a vascular accident could have resulted in complete separation of a segment from the classic duplication cyst resulting in the CIDC, as supported by the evidence that both the classic intraabdominal duplication cyst and the CIDC had similar epithelial lining of gastric mucosa (3).

Duplication cysts are best managed by early resection , as they have a tendency to cause lifethreatening complications such as bleeding, fistulization and even malignant degeneration ${ }^{(4,10)}$.With the advances in antenatal imaging, cystic lesions are now being diagnosed antenatally, which are best investigated by a postnatal CT scan(10).Awareness of varied presentations of duplications may help in appropriate management. An elective excision is safe with minimum morbidity and is the generally accepted surgical management (10).

In conclusion, our case demonstrates the benefits of antenatal detection, early investigations and successful elective excision of the cysts, which was curative. A careful search is warranted in all cases as there can be concomitant intraabdominal and intrathoracic duplication cysts associated with CIDC.

\section{REFERENCES:}

1. Birmole BJ, Kulkarni BK, Vaidya AS, Borwankar SS. Intrathoracic enteric foregut duplication cyst. J Postgrad Med 1994; 40:228.

2. R. I. Macpherson. Gastrointestinal tract duplications: clinical, pathologic, etiologic, and radiologic considerations. Radiographics 1993; 13(5):1063-1080.

3. Pant N, Grover JK, Madan NK, Chadha R, Agarwal K, Choudhury SR. Completely isolated enteric duplication cyst associated with a classic enterogenous duplication cyst. J Indian Assoc Pediatr Surg 2012;17(2):68-70

4. M. Gümüş, M. Kapan, H. Gümüş, A. Onder, and S. Girgin. Unusual noncommunicating isolated enteric duplication cyst in adults. Gastroenterology Research and Practice, vol. 2011, Article ID 323919, 3 pages, 2011.

5. Neofytou Kyriakos, Chysochos Andreas, Sammouti Elena, Andreou Charalampos, and Georgiou Chrisantho. Infected Completely Isolated Enteric Duplication Cyst Management with Percutaneous Drainage and Surgical Excision after Retreat of Infection: A Case Report. Case Reports in Surgery, vol. 2013, Article ID 108126, 4 pages, 2013.

6. HandaR, Kale R, Harjai MM, Synchronous Gut Duplication Cysts: A Rare Presentation. MJAFI 2007; 63: 376-377. 


\section{CASE REPORT}

7. Ringley C, Bochkarev V, Oleynikov D. Esophageal duplication cyst-A guest case in robotic and computer-assisted surgery from the University of Nebraska Medical Center. Med Gen Med 2006; 8(4):25.

8. Chaudhary S, Raju U, Harjai MM, Gupta M. Intestinal Duplication Cyst MJAFI 2006; 62: 87-88.

9. Nawaz A, Matta H, Jacobsz A, Ansari F, Khouder G A, Salem A. Intrathoracic foregut duplication cysts in neonates. Annals of Saudi Medicine 2001; 21:3-4.

10. Cocker D M, Parikh D and Brown R. Multiple Antenatally Diagnosed Foregut Duplication Cysts Excised and the Value of Thoracoscopy in Diagnosing Small Concurrent Cysts. Ann R Coll Surg Engl 2006; 88(6): 588.

Fig 1(a). Contrast enhanced CT scan abdomen-non enhancing cystic density mass lesion occupying the peritoneal cavity without septations/solid component.

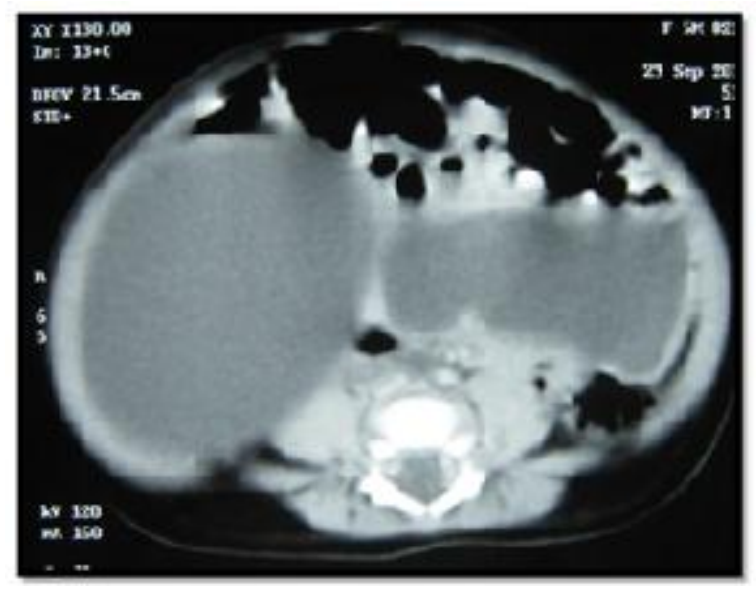

Fig 1(b). Contrast CT thorax-well defined peripherally enhancing cystic mass lesion involving posterior mediastinum in the right paravertebral region.

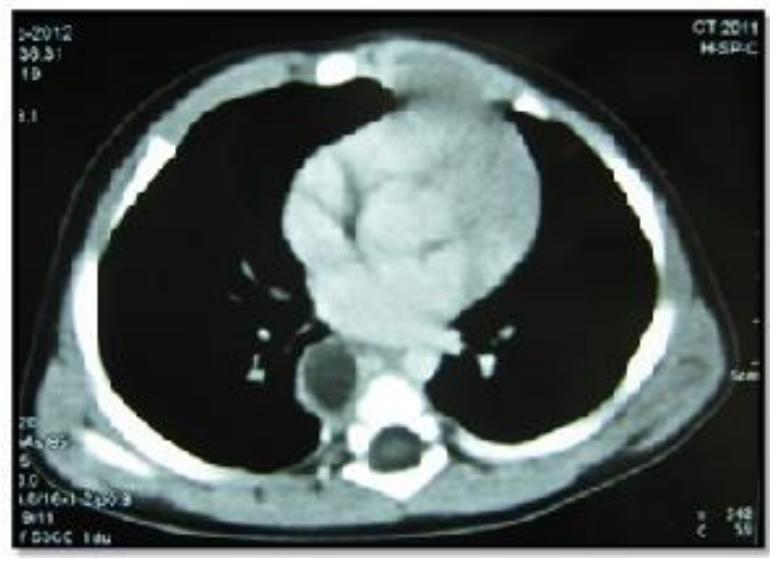




\section{CASE REPORT}

Fig 1(c). Per operative photograph showing dumbbell shaped ileal duplication cyst communicating through the mesentery

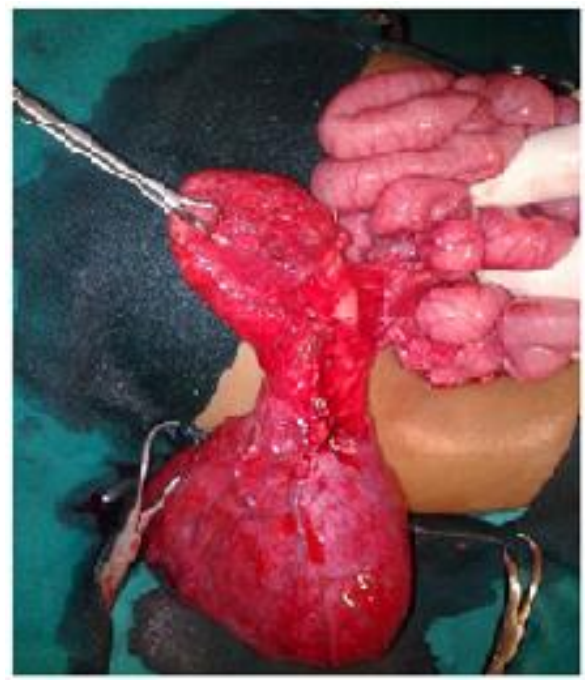

Fig 2(a). Ileal duplication cyst showing gastric mucosal lining and thick smooth muscle coats (H\&E, $\mathrm{X}$ 20).

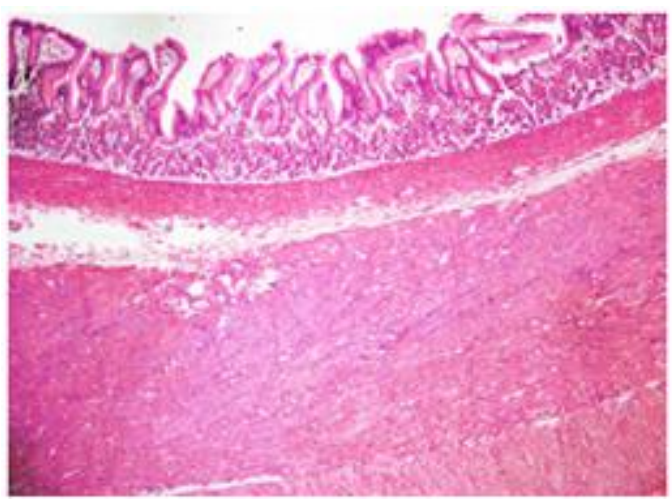

Fig 2(b). Gastric mucosal lining of ileal duplication cyst (H\&E, X 100)

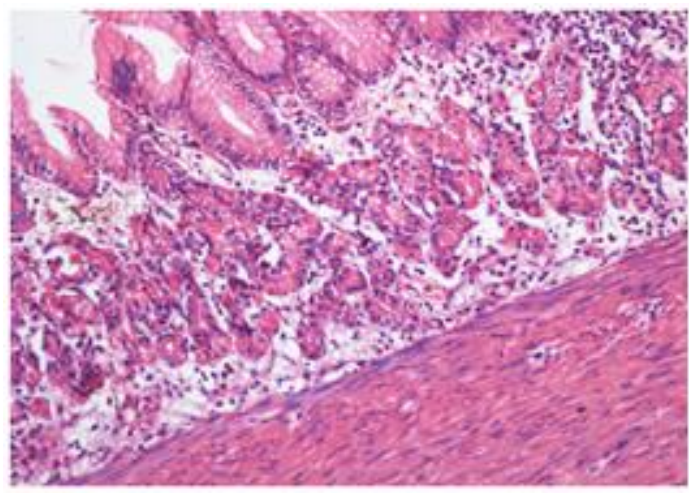




\section{CASE REPORT}

Fig 2(c). CIDC lined by pseudostratified ciliated columnar epithelium (H\&E, X 200)

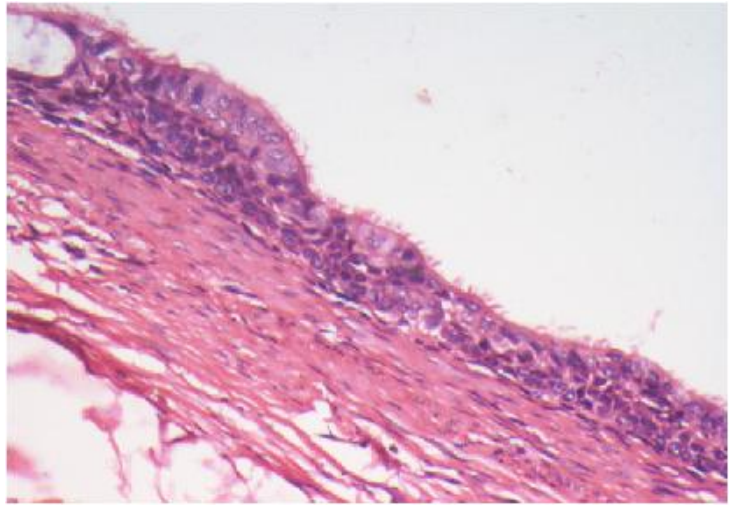

Fig 2(d). Oesophageal duplication cyst lined by gastric mucosa and stratified squamous epithelium (H\&E, X40)

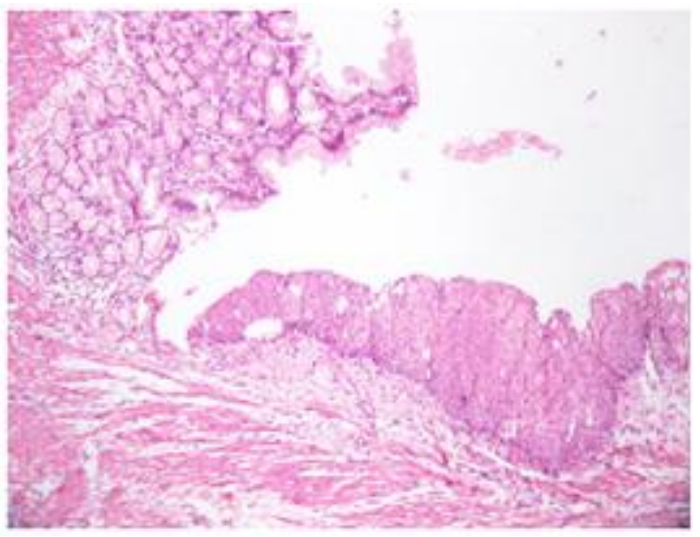

\section{AUTHORS:}

1. Rajalakshmi B.R.

2. Asha Mahadevappa.

3. Anilkumar M.G.

4. Sunila

5. Manjunath G.V.

\section{PARTICULARS OF CONTRIBUTORS:}

1. Assistant Professor, Department of Pathology, JSS Medical College, JSS University, Mysore, Karnataka, India.

2. Associate Professor, Department of Pathology, JSS Medical College, JSS University, Mysore, Karnataka, India.

3. Professor, Department of Paediatric Surgery, JSS Medical College, JSS University, Mysore, Karnataka, India.
4. Professor, Department of Pathology, JSS Medical College, JSS University, Mysore, Karnataka, India.

5. Professor \& HOD, Department of Pathology, JSS Medical College, JSS University, Mysore, Karnataka, India.

\section{NAME ADRRESS EMAIL ID OF THE CORRESPONDING AUTHOR:}

Dr. Rajalakshmi B.R, \#48, HPO \& RMS Layout, Behind RTO East, Shakthi Nagar, Mysore, Karnataka - 570029. Email - vishurad@gmail.com

Date of Submission: 29/07/2013. Date of Peer Review: 30/07/2013. Date of Acceptance: 06/08/2013. Date of Publishing: 10/08/2013. 